\title{
HIGHER RANK CLASS GROUPS ${ }^{1}$
}

\author{
BY LUTHER CLABORN AND ROBERT FOSSUM
}

Communicated by P. T. Bateman, October 24, 1966

Let $A$ be a noetherian ring which is locally Macaulay. For each in teger $i \geqq 0$, groups $C_{i}(A)$ and $W_{i}(A)$ are defined, each sequence of groups generalizing to higher dimensions the usual class group of an integrally closed noetherian domain. $C_{i}(A)$ is called the $i$ th class group of $A$, and $W_{i}(A)$ is called the $i$ th homological class group of $A$. The main purpose of this note is to show that both sequences of groups have properties analogous to the class group of a Noetherian integrally closed integral domain, and finally to establish a connection between them.

1. Throughout this section $A$ is a commutative noetherian ring which is locally Macaulay. A set of elements $x_{1}, \cdots, x_{8}$ is an $A$ sequence of length $s$ if $x_{1} A+\cdots+x_{s} A \neq A$ and $x_{1} A+\cdots+x_{i} A$ : $x_{i+1}=x_{1} A+\cdots+x_{i} A$ for $i=0,1, \cdots, s-1$. Count the empty set as an $A$-sequence of length 0 and specify that it generate the zero ideal of $A$.

Note that if $x_{1}, \cdots, x_{s}$ is an $A$-sequence of length $s$, then $x_{1} A$ $+\cdots+x_{8} A$ is an unmixed ideal of $A$ of height $s$.

For each $i \geqq 0$, form the free abelian group on the generators $\langle p\rangle$ where $\mathfrak{p}$ is a height $i$ prime ideal of $A$. This group will be denoted by $D_{i}(A)$. For each $A$-sequence $x_{1}, \cdots, x_{i}$, consider the element $\sum e\left(x_{1}, \cdots, x_{i} \mid A_{\mathfrak{p}}\right)\langle\mathfrak{p}\rangle$ of $D_{i}$ (here $e\left(y_{1}, \cdots, y_{i} \mid M\right)$ denotes the multiplicity of $y_{1} A+\cdots+y_{i} A$ on $M$ ). Let $R_{i}$ designate the subgroup of $D_{i}$ generated by all such elements. Set $C_{i}(A)=D_{i}(A) / R_{i}$ and call $C_{i}(A)$ the class group of rank $i$ for $A$. Denote the image of $\langle\mathfrak{p}\rangle$ in $C_{i}(A)$ by $\operatorname{cl}(\mathfrak{p})$. Set $C .(A)=\oplus C_{i}(A)$.

ExAmples. $C_{0}(A)$ is always finitely generated. $C_{0}(A)$ is finite if and only if (0) is a primary ideal of $A \cdot C_{0}(A)=0$ if and only if $A$ is a domain.

If $A$ is a Dedekind domain, then $C_{1}(A)$ is the ordinary ideal class group of $A$. More generally, if $A$ is integrally closed, then $C_{1}(A)$ is the class group of $A[1, \S 1$, no. 10$]$.

We have not been able to locate the following lemma in the literature.

1 This research was supported by the National Science Foundation Grant GP5478. 
Lemma 1.1. Let $S$ be a multiplicatively closed subset of $A$. If $y_{1}, \cdots$, $y_{i}$ is an $A_{S^{-}}$sequence, then there is an $A$-sequence $x_{1}, \cdots, x_{i}$ such that $\sum y_{i} A_{s}=\sum x_{i} A_{s}$.

Theorem 1.2. (Cf. [1, Proposition $17, \S 1$, no. 10].) Let $S$ be a multiplicatively closed subset of $A$. Then for each $i \geqq 0$, there is an epimorphism $C_{i}(A) \rightarrow C_{i}\left(A_{S}\right)$ deduced from $\langle\mathfrak{p}\rangle \rightarrow 0$ if $\mathfrak{p} \cap S \neq \varnothing$ and $\langle p\rangle \rightarrow\left\langle p A_{S}\right\rangle$ if $\mathfrak{p} \cap S=\varnothing$. The kernel is generated by $\{\operatorname{cl}(\mathfrak{p})\}$ where $h t(p)=i$ and $\mathrm{p} \cap S \neq \varnothing$.

Corollary 1.3. (Cf. [4, Lemma 1.7].) If $\mathfrak{p} \cap S \neq \varnothing$ implies that $\operatorname{cl}(p)=0$, then the epimorphism of Theorem 1.2 is an isomorphism.

Corollary 1.4. If $C_{i}\left(A_{s}\right)=0$, then $C_{i}(A)$ is generated by $\{\mathrm{cl}(p)\}$ where $h t(\mathfrak{p})=i$ and $\mathfrak{p} \cap S \neq \varnothing$.

Corollary 1.5. There is an epimorphism $C_{i}(A) \rightarrow \oplus_{h t(\mathfrak{p})=i} C_{i}\left(A_{\mathfrak{p}}\right)$ deduced from $\langle\mathfrak{p}\rangle \rightarrow\left\langle\mathfrak{p} A_{\mathfrak{p}}\right\rangle$.

TheOREM 1.6. If $x_{1}, \cdots, x_{k}$ is an $A$-sequence, then there is a homomorphism $C_{i}\left(A / \sum x_{s} A\right) \rightarrow C_{i+k}(A)$ whose image is the subgroup of $C_{i+k}(A)$ generated by $\{\mathrm{cl}(\mathfrak{p})\}$ where $h t(p)=i+k$ and $p \supseteq \sum x_{s} A$.

With Theorem 1.2, this yields

Corollary 1.7. Suppose that $x$ is an A-sequence. Then the sequence

$$
C_{i}(A / x A) \rightarrow C_{i+1}(A) \rightarrow C_{i+1}\left(A\left[x^{-1}\right]\right) \rightarrow 0
$$

is exact.

An application of the associative law for multiplicities yields

THEOREM 1.8. If $h t(\mathfrak{p})=k$ and $\mathrm{cl}(\mathfrak{p})=0$, then there is a homomorphism $C_{i}(A / \mathfrak{p}) \rightarrow C_{i+k}(A)$ whose image is the subgroup of $C_{i+k}(A)$ generated by the $\operatorname{cl}(\mathfrak{q})$ where $h t(\mathfrak{q})=i+k$ and $\mathfrak{q} \supseteq \mathfrak{p}$.

Using techniques similar to those of [2, Proof of Proposition 7-1] we get

Lemma 1.9. Suppose that $C_{i}\left(A_{\mathfrak{p}}\right)=0$ for each prime ideal $\mathfrak{p}$ of height $i$ of $A$. Then $C_{i+1}(A[X])$ is generated by $\{\operatorname{cl}(\mathfrak{q} A[X])\}$ where $\mathfrak{q}$ ranges over the prime ideals of $A$ of height $i+1$.

Theorem 1.10. If $C_{i}\left(A_{\mathfrak{p}}\right)=0$ for all prime ideals $\mathfrak{p}$ of $A$ of height $i$, then there is an epimorphism $C_{i+1}(A) \rightarrow C_{i+1}(A[X])$.

Corollary 1.11. (Cf. [1, Corollary to Theorem 2].) $C .(A)=0$ implies $C .(A[X])=0$. 
REMARK. Corollary 1.11 does not hold for power series adjunction as Samuel's example [4] shows.

Corollary 1.12. If $F$ is a field, then $C \cdot\left(F\left[X_{1}, \cdots, X_{n}\right]\right)=0$.

Corollary 1.13. Let the Krull dimension of $A$ be $n<\infty$. Suppose that $C_{n}\left(A_{\mathfrak{p}}\right)=0$ for each prime ideal $\mathfrak{p}$ of $A$ of height $n$. Then $C_{n+1}(A[X])=0$.

A theorem similar to Theorem 1.10 is

Theorem 1.14. Let $A$ and $B$ be finitely generated over a field $F$. Suppose, that for each $i \geqq 0, C_{i}\left(A_{\mathfrak{p}}\right)=0$ for any prime ideal $\mathfrak{p}$ of height $i$ of $A$, and that $C .\left(K \otimes_{F} B\right)=0$ for any overfield $K$ of $F$. Then there is an epimorphism $C_{j}(A) \rightarrow C_{j}\left(A \otimes_{F} B\right)$ given by $\mathrm{cl}(p) \rightarrow \operatorname{cl}\left(p \otimes_{F} B\right)$. In particular, $C .(A)=0$ implies $C .\left(A \otimes_{F} B\right)=0$.

Theorem 1.15. For $i \geqq 1, C_{i}\left(A_{1} \oplus A_{2}\right)=C_{i}\left(A_{1}\right) \oplus C_{i}\left(A_{2}\right)$.

2. Let $A$ be a commutative noetherian ring. The hypotheses on $A$ in $\$ 1$ need not be assumed in order to define the groups $W_{i}(A)$. The reader is referred to $[3]$ for the $K$-theory needed here.

Let $\mathfrak{T}_{i}(A)=\mathfrak{M}_{i}$ denote the category of finitely generated $A$ modules $M$ such that $M_{\mathfrak{p}}=0$ for all prime ideals $\mathfrak{p}$ of $A$ with $h t(\mathfrak{p})<i$. Then $\mathfrak{M}_{j}$ is a Serre subcategory of $\mathfrak{T}_{i}$ for all $j>i$. Let $K^{i}(\mathcal{C})$ denote the $i$ th Grothendieck group of the category $\mathfrak{e}$ for $i=0,1$. If $C \in \mathbb{e}$, then $\gamma(C)$ denotes the image (or class) of $C$ in $K^{0}(\mathcal{e})$.

Proposition 2.1. $K^{0}\left(\mathfrak{T H}_{i} / \mathfrak{T}_{i+1}\right)$ is isomorphic to $D_{i}(A)$, the isomorphism being given by the length function.

Consider the following commutative diagram

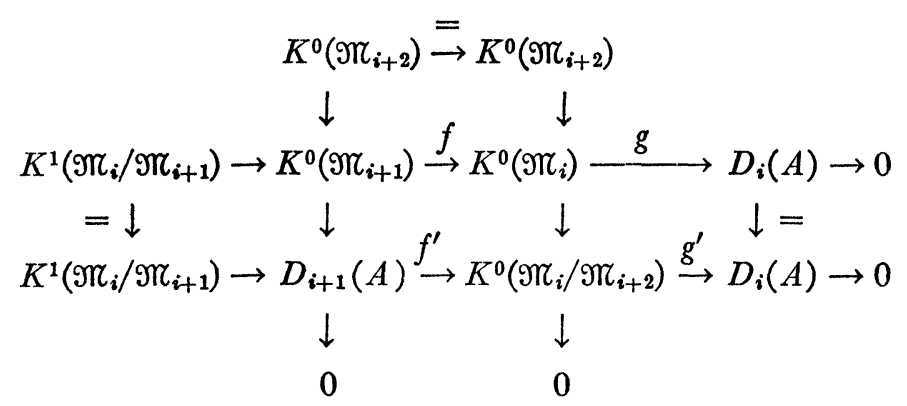

Because each element in $\mathfrak{T K}_{i} / \mathfrak{T K}_{i+1}$ has finite length, each of the rows is exact. The columns are also exact.

Since the group $D_{i}(A)$ is free, the kernels of $g$ and $g^{\prime}$ in the above diagram are direct summands of their respective domains. For each 
$i \geqq 0$ define the group $Z_{i+1}(A)$, and the homological class group of rank $i+1, W_{i+1}(A)$, to be the kernels of $g$ and $g^{\prime}$ respectively. Since the rows are exact this is the same as saying that $Z_{i+1}(A)$ is the image of $f$ and $W_{i+1}(A)$ is the image of $f^{\prime}$. Moreover

$$
K^{0}\left(\mathfrak{T T}_{i}\right)=Z_{i+1}(A) \oplus D_{i}(A)
$$

and

$$
K^{0}\left(\mathfrak{T K}_{i} / \mathfrak{T K}_{i+2}\right)=W_{i+1}(A) \oplus D_{i}(A) .
$$

The results of [3] yield

Proposition 2.2. $K^{1}\left(\mathfrak{N T}_{i} / \mathfrak{M T}_{i+1}\right)$ is isomorphic to the direct sum of the groups of units of $A_{\mathfrak{p}} / \mathfrak{p} A_{\mathfrak{p}}, h t(\mathfrak{p})=i$. Consequently the kernel of $f^{\prime}$ is generated by the $\gamma(A /(\mathfrak{p}+x A)), x \notin \mathfrak{p}$, as $\mathfrak{p}$ ranges over the prime ideals of $A$ of height $i$, and hence $W_{i+1}(A)$ is $D_{i+1}(A)$ modulo the subgroup generated by these.

By convention $W_{0}(A)=0$. Set $W \cdot(A)=\oplus W_{i}(A)$.

Diagram chasing will give

TheOREM 2.3. Let $S$ be a multiplicatively closed subset of $A$. For each $i$ there is an epimorphism $W_{i}(A) \rightarrow W_{i}\left(A_{S}\right)$ induced by the functor $A_{S} \otimes_{A}-$. The kernel is generated by $\gamma(A / \mathfrak{p})$ with $\mathfrak{p} \cap S \neq \varnothing, h t(\mathfrak{p})=i$.

Corollary 2.4. If for each prime ideal $\mathfrak{p}$ of $A$ of height $i$ with $\mathfrak{p} \cap S \neq \varnothing, \gamma(A / \mathfrak{p})=0$ in $K^{0}\left(\mathscr{T}_{i-1} / \mathscr{M}_{i+1}\right)$ then the epimorphism of Theorem 2.3 is an isomorphism.

Corollary 2.5. If $W_{i}\left(A_{S}\right)=0$, then $W_{i}(A)$ is generated by $\{\gamma(A / \mathfrak{p})\}$, $h t(\mathfrak{p})=i, \mathfrak{p} \cap S \neq \varnothing$.

Corollary 2.6. The functors $A_{p} \otimes_{A}$-induce an epimorphism $W_{i}(A)$ $\rightarrow \bigoplus_{h t(\mathfrak{p})=i} W_{i}\left(A_{\mathfrak{p}}\right)$.

Theorem 2.7. Let $A$ be locally Macaulay, I an unmixed ideal of height $k$. Then there is a homomorphism

$$
W_{i}(A / I) \rightarrow W_{i+k}(A)
$$

induced by considering each $A / I$-module as an $A$-module. The image is generated by the $\gamma(A / \mathfrak{p}), \mathfrak{p}$ a prime ideal of height $i+k$ containing $I$.

Using Theorems 2.3 and 2.7 one gets

Theorem 2.8. Let $x$ be an A-sequence, $A$ a locally Macaulay ring. Then the sequence 


$$
W_{i}(A / x A) \rightarrow W_{i+1}(A) \rightarrow W_{i+1}\left(A\left[x^{-1}\right]\right) \rightarrow 0
$$

is exact.

THEOREM 2.8. The functor $A[X] \otimes_{A}$-induces an epimorphism $W_{i}(A)$ $\rightarrow W_{i}(A[X])$. Furthermore $W_{n+1}(A[X])=0$ if the Krull dimension of $A$ is $n<\infty$.

Corollary 2.9. $W \cdot(A)=0$ implies $W \cdot(A[X])=0$.

Corollary 2.10. $W \cdot\left(F\left[X_{1}, \cdots, X_{n}\right]\right)=0$ when $F$ is a field.

Theorem 2.11. Let $A_{1}$ and $A_{2}$ be two rings. Then

$$
W_{i}\left(A_{1} \oplus A_{2}\right)=W_{i}\left(A_{1}\right) \oplus W_{i}\left(A_{2}\right) \text {. }
$$

3. It is natural to ask if $C_{i}(A)=W_{i}(A)$ when both are defined. There are several results in this direction.

THEOREM 3.1. $W_{i}(A)$ is a homomorphic image of $C_{i}(A)$.

Theorem 3.2. If $C_{i}(A)=0$, then $W_{i+1}(A)=C_{i+1}(A)$.

Corollary 3.3. If $A$ is a domain, then $W_{1}(A)=C_{1}(A)$.

Corollary 3.4. $C \cdot(A)=0$ if, and only if, $A$ is an integral domain and $W \cdot(A)=0$.

For an example which shows that in general $W \cdot(A) \neq C \cdot(A)$ let $Q$ be a primary ring which is not a field and set $A=Q[X]$. Then $W_{1}(A)=0$ while $C_{1}(A)$ is an infinite group.

\section{REFERENCES}

1. N. Bourbaki, Algebre commutative, Chapitre 7, Hermann, Paris, 1965.

2. L. Claborn, On the theory of E-rings, Doctoral Dissertation, University of Michigan, Ann Arbor, Michigan, 1963. 409.

3. A. Heller, Some exact sequences in algebraic K-theory, Topology 3 (1965), 389-

4. P. Samuel, On unique factorization domains, Illinois J. Math. 5 (1961), 1-17.

UNIVERSITY OF ILLINOIS, URBANA 